\title{
Trajectory Control of Rendezvous with Maneuver Target Spacecraft
}

\author{
Zhiqiang Zhou ${ }^{1}$ \\ NASA Langley Research Center, Hampton, VA 23681
}

\begin{abstract}
In this paper, a nonlinear trajectory control algorithm of rendezvous with maneuvering target spacecraft is presented. The disturbance forces on the chaser and target spacecraft and the thrust forces on the chaser spacecraft are considered in the analysis. The control algorithm developed in this paper uses the relative distance and relative velocity between the target and chaser spacecraft as the inputs. A general formula of reference relative trajectory of the chaser spacecraft to the target spacecraft is developed and applied to four different proximity maneuvers, which are in-track circling, cross-track circling, in-track spiral rendezvous and cross-track spiral rendezvous. The closed-loop differential equations of the proximity relative motion with the control algorithm are derived. It is proven in the paper that the tracking errors between the commanded relative trajectory and the actual relative trajectory are bounded within a constant region determined by the control gains. The prediction of the tracking errors is obtained. Design examples are provided to show the implementation of the control algorithm. The simulation results show that the actual relative trajectory tracks the commanded relative trajectory tightly. The predicted tracking errors match those calculated in the simulation results. The control algorithm developed in this paper can also be applied to interception of maneuver target spacecraft and relative trajectory control of spacecraft formation flying.
\end{abstract}

\section{Introduction}

$\mathrm{T}$ RAJECTORY control of spacecraft rendezvous has been studied for many years. For the most of the studies, the target spacecraft doesn't maneuver by firing thrusters during the rendezvous [1-5]. Using the linearized Tschauner-Hempel equations, spacecraft rendezvous with target spacecraft in an arbitrary elliptical orbit was analyzed [1]. The constrained regulation problem is solved based on a parametric Lyapunov differential equation approach. The disturbance forces on the chaser and target spacecraft and the thrust forces on the chaser spacecraft are not considered in the analysis in [1]. The six-degree-of-freedom elliptical orbit rendezvous and docking problem were investigated using an adaptive discrete-time robust nonlinear filtering technique [2]. However, the disturbance and thrust forces on the target spacecraft are neglected in the relative motion dynamics in [2].

In this paper, a nonlinear trajectory control algorithm of rendezvous with maneuvering target spacecraft is presented. The relative motion equation of the maneuvering target spacecraft and chaser spacecraft is developed. The acceleration due to thrust forces on the target spacecraft for maneuvers is included in the equation. The disturbance forces, such as aerodynamic drag and solar pressure are also considered in the equation. The relative motion is governed by a nonlinear time variant differential equation.

The target spacecraft orbit variation, due to its maneuvers, imposes a challenge for the control system design. The control algorithm developed in this paper uses the relative distance and relative velocity between the target and chaser spacecraft as the inputs. The relative distance and relative velocity can be measured by a lidar sensor. A reference relative trajectory of the chaser spacecraft to the target spacecraft are formed based on the required rendezvous, such as approaching the target spacecraft and circling it or approaching the target spirally and docking with it eventually. The reference relative trajectory can be in the in-track plan of the target spacecraft or the crosstrack plan. The thrust command output from the control system is calculated based on the differences of the relative distance and the reference relative trajectory as well as the measured relative velocity.

Several proximity and rendezvous maneuvers are realized using the control algorithm described in this paper. The first proximity maneuver is in-track circling, in which the chaser spacecraft circulates the target spacecraft at a

${ }^{1}$ Aerospace Technologist, NASA Langley Research Center, M/S 488, 5 N. Dryden St., Hampton, VA 23681. zhiqiang.zhou@nasa.gov, Senior Member AIAA. 
circular orbit relative to the target with a constant rate. The relative circular orbit is in the target spacecraft's intrack plan. Similarly the cross-track circling is to circulate the target spacecraft in the target spacecraft's cross-track plan. In the next two rendezvous maneuvers the chaser spacecraft approaches the target spacecraft along a spiral trajectory in the target spacecraft's in-track or cross-track plans. Design examples and simulation results are presented at the end of the paper to demonstrate the performance of the control algorithm. The control algorithm developed in this paper can be applied to interception of maneuver target spacecraft and relative trajectory control of spacecraft formation flying also.

\section{Proximity Relative Motion Model}

The proximity relative motion model of chaser spacecraft relative to target spacecraft has to be established before the rendezvous trajectory control algorithms can be developed. In Figure 1, the target spacecraft orbit frame $x_{o t}, y_{o t}, z_{o t}$ and the chaser spacecraft orbit frame $x_{o c}, y_{o c}, z_{o c}$ are depicted. The trajectory dynamics for the target spacecraft in the inertial frame is

$$
\ddot{R}=-\mu \frac{R}{|R|^{3}}+a_{d t}+a_{t}
$$

$R$ is the distance vector from the center of gravity to the target spacecraft and $\mu$ is the gravitational parameter of the planet. $a_{d t}$ is the target spacecraft acceleration with respect to (wrt) the inertial frame due to the disturbance forces, such as aerodynamic drag, solar pressure. $a_{t}$ is the target spacecraft acceleration wrt the inertial frame due to the spacecraft thrust forces.

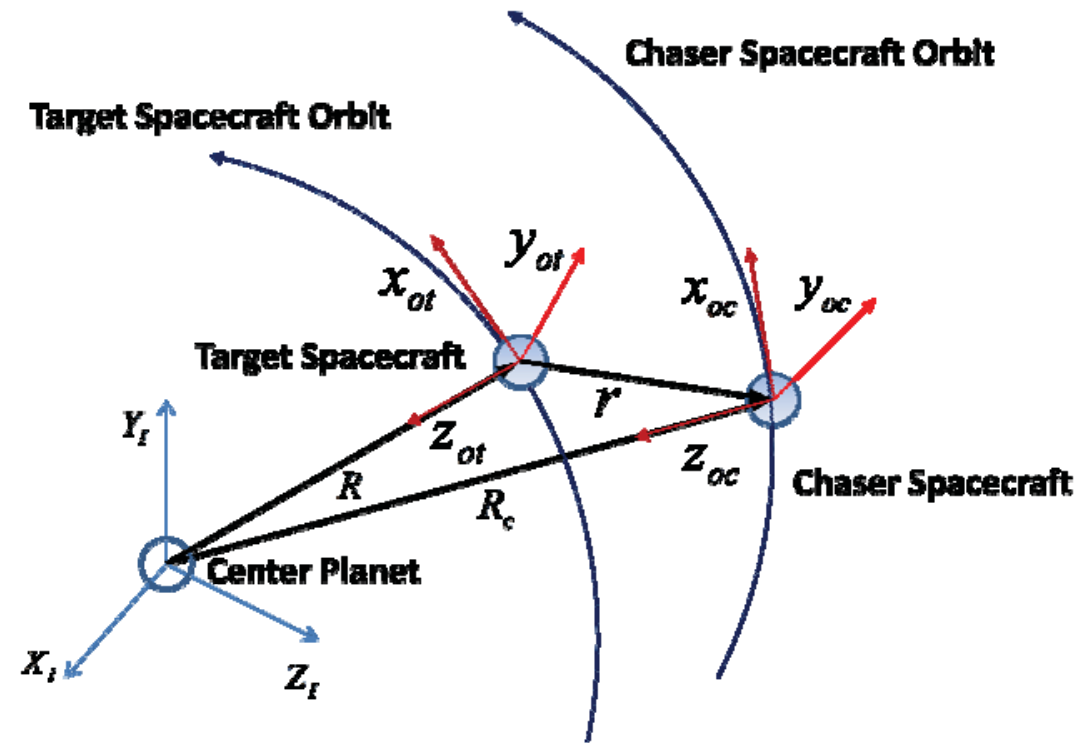

Figure 1. Spacecraft redezvous geometry and coordinates.

The equation of motion of the chaser spacecraft in the inertial frame is given by

$$
\ddot{R}_{c}=\ddot{R}+\ddot{r}=-\mu \frac{R+r}{|R+r|^{3}}+a_{d c}+a_{c}
$$

$r$ is the relative distance vector from the target spacecraft to the chaser spacecraft. $a_{d c}$ is the target spacecraft acceleration wrt the inertial frame due to the disturbance forces. $a_{c}$ is the target spacecraft acceleration wrt the inertial frame due to the thrust forces. Using Eq.(1) and Eq.(2), the differential equation of the relative motion can be obtained as 


$$
\ddot{r}=\mu\left(\frac{R}{|R|^{3}}-\frac{R+r}{|R+r|^{3}}\right)+a_{d c}+a_{c}-a_{d t}-a_{t}
$$

Note that the above equation has simple form because the differential equation is written in the inertial frame $X_{I}$, $Y_{I}, Z_{I}$. In general, the disturbance forces and the thrust forces are calculated in the spacecraft body frame. The accelerations in the inertial frame can be obtained by transform the disturbance forces and the thrust forces from the body frame to the inertial frame as

$$
\begin{aligned}
& a_{d t}=\frac{A_{b t}^{I} F_{d t}}{m_{t}} \\
& a_{t}=\frac{A_{b t}^{I} T_{t}}{m_{t}} \\
& a_{d c}=\frac{A_{b c}^{I} F_{d c}}{m_{c}} \\
& a_{c}=\frac{A_{o t}^{I} T_{c}}{m_{c}}
\end{aligned}
$$

$A_{b t}^{I}$ is the transformation matrix from the target spacecraft body frame to the inertial frame. $A_{b c}^{I}$ is the transformation matrix from the chaser spacecraft body frame to the inertial frame. $F_{d t}$ is the disturbance forces on the target spacecraft wrt the target spacecraft body frame. $m_{t}$ and $m_{c}$ are the target and chaser spacecraft mass respectively. $T_{t}$ is the target spacecraft thrust forces wrt its own body frame. However, $T_{c}$ is the chaser spacecraft thrust forces wrt the target spacecraft orbit frame $x_{o t}, y_{o t}, z_{o t}$ because the rendezvous trajectory control algorithms are formed in the target spacecraft orbit frame as described in the next section. The chaser spacecraft thrust forces wrt its own body frame $T_{b c}$ can be calculated by

$$
T_{b c}=A_{o t}^{b c} T_{c}
$$

Eq.(3) describes the relative motion between the target and the chaser spacecraft. No simplification and approximation are made in Eq.(3). The equation includes all the forces acting on the target and the chaser spacecraft. The target spacecraft can perform any maneuver by firing the thrusters.

\section{Trajectory Control Algorithms and Performance}

This section develops the trajectory control algorithm to control the chaser spacecraft relative motion to the target spacecraft. The control algorithm calculates the chaser spacecraft thrust forces wrt the target spacecraft orbit frame $T_{c}$ as

$$
T_{c}=m_{c}\left\{K_{r}\left(r_{c m d}-A_{I}^{o t} r\right)+K_{v}\left(\dot{r}_{c m d}-A_{I}^{o t} \dot{r}\right)+\ddot{r}_{c m d}\right\}
$$

$K_{r}$ and $K_{v}$ are control gains, which can be matrix or scalar. $r$ is the relative distance vector from the target spacecraft to the chaser spacecraft expressed in the inertial frame. $A_{I}^{o t}$ is the transformation matrix from the inertial frame to the target spacecraft orbit frame, which transforms $r$ to the orbit frame. $\dot{r}$ is the relative velocity, which is the derivative of the relative distance vector wrt the inertial frame. The relative distance and relative velocity can be measured by a lidar sensor. $r_{c m d}$ is the commanded reference relative distance, which is formed based on the type of the relative motions, such as approaching the target spacecraft and circling it or approaching the target spirally and docking with it eventually. $\dot{r}_{c m d}$ is the commanded relative velocity, which is the derivative of the commanded relative distance vector wrt the target orbit frame. $\ddot{r}_{c m d}$ is the derivative of $\dot{r}_{c m d}$ wrt the target orbit frame.

$r_{c m d}$ can be determined in the target orbit frame by the azimuth angle $\alpha$ and the elevation angle $\beta$ as shown in Figure 2. The commanded reference relative distance is given by 


$$
r_{c m d}(t) \equiv\left(\begin{array}{c}
r_{x c m d}(t) \\
r_{y c m d}(t) \\
r_{z c m d}(t)
\end{array}\right)=\left(\begin{array}{c}
r_{m}(t) \cos \alpha(t) \cos \beta(t) \\
r_{m}(t) \sin \alpha(t) \cos \beta(t) \\
r_{m}(t) \sin \beta(t)
\end{array}\right)
$$

$r_{m}(t)$ is the magnitude of the commanded relative distance. $\alpha$ and $\beta$ depend on the desired relative motions. In the following expression $\alpha$ and $\beta$ are changing in constant rates $\omega_{\alpha}$ and $\omega_{\beta}$ with the time.

$$
\left\{\begin{array}{l}
\alpha(t)=\alpha_{0}+\omega_{\alpha} t \\
\beta(t)=\beta_{0}+\omega_{\beta} t
\end{array}\right.
$$

The initial values of $\alpha$ and $\beta$ are determined such that the commanded relative distance is equal to the actual relative distance at the beginning in order to make the control smooth.

$$
r_{c m d}(0)=A_{I}^{o t} r(0)
$$

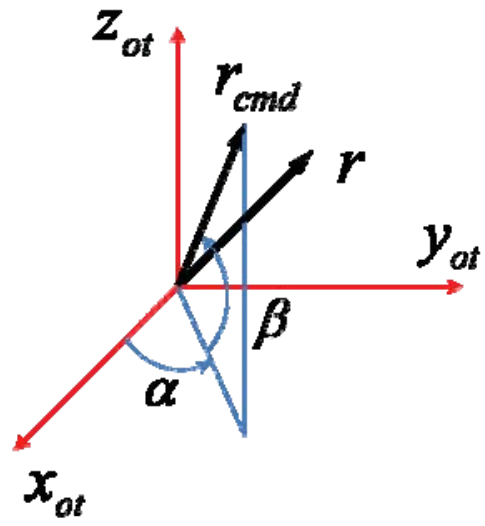

Figure 2. Commanded relative trajectory in the target spacecraft orbit frame

The commanded reference trajectories for several proximity maneuvers are developed. The first proximity maneuver is in-track circling, in which the chaser spacecraft circulates the target spacecraft at a circular orbit relative to the target with a constant rate. The relative circular orbit is in the target spacecraft's in-track (x-z) plan. The commanded reference trajectory is given by

$$
\begin{aligned}
& r_{c m d}(t)=\left(\begin{array}{c}
r_{m} \sin \left(\omega t+\theta_{0}\right) \\
0 \\
-r_{m} \cos \left(\omega t+\theta_{0}\right)
\end{array}\right) \\
& \dot{r}_{c m d}(t)=\left(\begin{array}{c}
r_{m} \omega \cos \left(\omega t+\theta_{0}\right) \\
0 \\
r_{m} \omega \sin \left(\omega t+\theta_{0}\right)
\end{array}\right) \\
& \ddot{r}_{c m d}(t)=\left(\begin{array}{c}
-r_{m} \omega^{2} \sin \left(\omega t+\theta_{0}\right) \\
0 \\
r_{m} \omega^{2} \cos \left(\omega t+\theta_{0}\right)
\end{array}\right)
\end{aligned}
$$

The magnitude of the commanded relative distance $r_{m}$ is constant for the in-track circling orbit. $\omega$ is the constant circling orbit rate. $\theta_{0}$ is determined by Eq.(12). Similarly the cross-track circling is to circulate the target spacecraft in the target spacecraft's cross-track (y-z) plan. The commanded reference trajectory can be expressed as 


$$
\begin{aligned}
& r_{c m d}(t)=\left(\begin{array}{c}
0 \\
r_{m} \sin \left(\omega t+\theta_{0}\right) \\
-r_{m} \cos \left(\omega t+\theta_{0}\right)
\end{array}\right) \\
& \dot{r}_{c m d}(t)=\left(\begin{array}{c}
0 \\
r_{m} \omega \cos \left(\omega t+\theta_{0}\right) \\
r_{m} \omega \sin \left(\omega t+\theta_{0}\right)
\end{array}\right) \\
& \ddot{r}_{c m d}(t)=\left(\begin{array}{c}
0 \\
-r_{m} \omega^{2} \sin \left(\omega t+\theta_{0}\right) \\
r_{m} \omega^{2} \cos \left(\omega t+\theta_{0}\right)
\end{array}\right)
\end{aligned}
$$

In the next two rendezvous maneuvers the chaser spacecraft approaches the target spacecraft along a spiral trajectory in the target spacecraft's in-track or cross-track plans. For in-track spiral rendezvous the commanded reference trajectory is

$$
\begin{aligned}
& r_{c m d}(t)=\left(\begin{array}{c}
r_{m}(t) \sin \left(\omega t+\theta_{0}\right) \\
0 \\
-r_{m}(t) \cos \left(\omega t+\theta_{0}\right)
\end{array}\right) \\
& \dot{r}_{c m d}(t)=\left(\begin{array}{c}
\dot{r}_{m}(t) \sin \left(\omega t+\theta_{0}\right)+r_{m}(t) \omega \cos \left(\omega t+\theta_{0}\right) \\
0 \\
-\dot{r}_{m}(t) \cos \left(\omega t+\theta_{0}\right)+r_{m}(t) \omega \sin \left(\omega t+\theta_{0}\right)
\end{array}\right) \\
& \ddot{r}_{c m d}(t)=\left(\begin{array}{c}
2 \dot{r}_{m}(t) \omega \cos \left(\omega t+\theta_{0}\right)-r_{m}(t) \omega^{2} \sin \left(\omega t+\theta_{0}\right) \\
0 \\
2 \dot{r}_{m}(t) \omega \sin \left(\omega t+\theta_{0}\right)+r_{m}(t) \omega^{2} \cos \left(\omega t+\theta_{0}\right)
\end{array}\right) \\
& r_{m}(t)=\left\{\begin{array}{cc}
r_{m i} & t<t_{i} \\
r_{m f} & t>t_{f} \\
r_{m i}-\left(r_{m i}-r_{m f}\right)\left(t-t_{i}\right) /\left(t_{f}-t_{i}\right) & t_{i} \leq t \leq t_{f}
\end{array}\right. \\
& \dot{r}_{m}(t)=\left\{\begin{array}{cc}
0 & t<t_{i} \quad \text { or } \quad t>t_{f} \\
-\left(r_{m i}-r_{m f}\right) /\left(t_{f}-t_{i}\right) & t_{i} \leq t \leq t_{f}
\end{array}\right.
\end{aligned}
$$

$t_{i}$ is the starting time of the spiral and $t_{f}$ is the ending time. $r_{m i}$ is the initial commanded relative distance and $r_{m f}$ is the distance at the ending time. For rendezvous $r_{m f}$ is zero. Similarly, the commands for the cross-track spiral rendezvous are given by

$$
\begin{aligned}
& r_{c m d}(t)=\left(\begin{array}{c}
0 \\
r_{m}(t) \sin \left(\omega t+\theta_{0}\right) \\
-r_{m}(t) \cos \left(\omega t+\theta_{0}\right)
\end{array}\right) \\
& \dot{r}_{c m d}(t)=\left(\begin{array}{c}
0 \\
\dot{r}_{m}(t) \sin \left(\omega t+\theta_{0}\right)+r_{m}(t) \omega \cos \left(\omega t+\theta_{0}\right) \\
-\dot{r}_{m}(t) \cos \left(\omega t+\theta_{0}\right)+r_{m}(t) \omega \sin \left(\omega t+\theta_{0}\right)
\end{array}\right)
\end{aligned}
$$




$$
\ddot{r}_{c m d}(t)=\left(\begin{array}{c}
0 \\
2 \dot{r}_{m}(t) \omega \cos \left(\omega t+\theta_{0}\right)-r_{m}(t) \omega^{2} \sin \left(\omega t+\theta_{0}\right) \\
2 \dot{r}_{m}(t) \omega \sin \left(\omega t+\theta_{0}\right)+r_{m}(t) \omega^{2} \cos \left(\omega t+\theta_{0}\right)
\end{array}\right)
$$

$r_{m}(t)$ and $\dot{r}_{m}(t)$ are determined by Eq.(22) and Eq.(23).

When the control algorithm in Eq.(9) is substituted into Eq.(3), the closed-loop differential equations of the proximity relative motion in the inertial frame is given by

$$
\ddot{r}=A_{o t}^{I}\left\{K_{r}\left(r_{c m d}-A_{I}^{o t} r\right)+K_{v}\left(\dot{r}_{c m d}-A_{I}^{o t} \dot{r}\right)+\ddot{r}_{c m d}\right\}+\mu\left(\frac{R}{|R|^{3}}-\frac{R+r}{|R+r|^{3}}\right)+\frac{A_{b c}^{I} F_{d c}}{m_{c}}-\frac{A_{b t}^{I} F_{d t}}{m_{t}}-\frac{A_{b t}^{I} T_{t}}{m_{t}}
$$

In general, Eq.(27) is a 3 dimensional nonlinear time variant differential equation. In order to measure the performance of the algorithm, the difference between the commanded relative trajectory $r_{c m d}$ and the actual relative trajectory $r$ is defined as $\lambda$, which is given by

$$
\lambda=r_{c m d}-A_{I}^{o t} r
$$

Eq.(27) can be rewritten in terms of $\lambda$ as

$$
\ddot{\lambda}+K_{v} \dot{\lambda}+K_{r} \lambda=f(t)
$$

with

$$
\begin{aligned}
& f(t) \equiv\left(f_{x}(t) \quad f_{y}(t) \quad f_{z}(t)\right)^{T} \\
& =A_{I}^{o t}\left\{\frac{A_{b t}^{I} F_{d t}}{m_{t}}+\frac{A_{b t}^{I} T_{t}}{m_{t}}-\mu\left(\frac{R}{|R|^{3}}-\frac{R+r}{|R+r|^{3}}\right)-\frac{A_{b c}^{I} F_{d c}}{m_{c}}\right\}-\left(K_{v}+\Omega_{o t}\right) \Omega_{o t} A_{I}^{o t} r-2 \Omega_{o t} A_{I}^{o t} \dot{r}
\end{aligned}
$$

The rotation rate vector $\omega_{o t}$ of the target spacecraft orbit frame $x_{o t}, y_{o t}, z_{o t}$ can be written as

$$
\omega_{o t}=\left(\begin{array}{lll}
\omega_{x o t} & \omega_{y o t} & \omega_{z o t}
\end{array}\right)^{T}
$$

Matrix $\Omega_{o t}$ is given by

$$
\Omega_{o t}=\left(\begin{array}{ccc}
0 & \omega_{z o t} & -\omega_{y o t} \\
-\omega_{z o t} & 0 & \omega_{x o t} \\
\omega_{y o t} & -\omega_{x o t} & 0
\end{array}\right)
$$

If the gain matrixes $K_{v}$ and $K_{r}$ are positive definite, and $f(t)$ is zero, it can be proved that $\lambda=0$ is a globally asymptotically stable equilibrium point of Eq.(29). It means that the actual relative trajectory $r$ will track the commanded relative trajectory $r_{c m d}$ and eventually converges to $r_{c m d}$. Usually $f(t)$ is not zero especially when the target spacecraft maneuvers during the chaser spacecraft approaching. However, $f(t)$ can not be unlimited because the accelerations due to the target spacecraft thrust and the disturbance forces are limited. If $f(t)$ satisfies the bound

$$
\left\|f_{i}(t)\right\| \leq f_{i \max } \quad(i=x, y, z)
$$

with

$$
f_{\max } \equiv\left(\begin{array}{lll}
f_{x \max } & f_{y \max } & f_{z \max }
\end{array}\right)^{T}
$$

Then it can be proved that $\lambda$ is globally uniformly ultimately bounded as

$$
\|\lambda\| \leq\left\|K_{r}^{-1} f_{\max }\right\|
$$


as time approaches infinity. The tracking error of the close-loop system with the control algorithm converges and is bounded to $\left\|K_{r}^{-1} f_{\max }\right\|$. The equation shows that increasing the gain matrix $K_{r}$ can reduce the final tracking error. Eq.(35) can be used to estimate the final errors between the actual relative trajectory and the commanded relative trajectory. The estimate by Eq.(35) is consistent with the simulation results as shown in the next section.

\section{Design Examples}

In order to evaluate the performance of the control algorithm developed in this paper, two rendezvous maneuvers are demonstrated. In these two maneuvers, the chaser spacecraft approaches the target spacecraft along a spiral trajectory in the target spacecraft's in-track or cross-track plans. The target spacecraft orbit is $488 \times 528 \mathrm{~km}$ with 72 degrees inclination. The target and chaser spacecraft masses are 600.0 and $400.0 \mathrm{Kg}$ respectively.

The initial relative distance $r(0)$ between the target and the chaser spacecraft is set to 10 meters. $r(0)$ wrt the target orbit frame is

$$
A_{I}^{o t} r(0)=\left(\begin{array}{lll}
0 & 0 & -10
\end{array}\right)^{T}
$$

During the rendezvous the target spacecraft conducts maneuvers by firing the thrusters in all three directions. Suppose that the thrust forces in in-track, cross-track and radial direction of the target orbit frame is sine waves and given by

$$
A_{b t}^{o t} T_{t}=\left(\begin{array}{l}
T_{x} \sin \left(2 \pi t / t_{x}+\theta_{x}\right) \\
T_{y} \sin \left(2 \pi t / t_{y}+\theta_{y}\right) \\
T_{z} \sin \left(2 \pi t / t_{z}+\theta_{z}\right)
\end{array}\right)
$$

The parameters in the above expression have the following values in the simulations.

$$
\begin{aligned}
& T_{x}=2.0(\mathrm{~N}) \quad T_{y}=4.0(\mathrm{~N}) \quad T_{z}=5.0(\mathrm{~N}) \\
& t_{x}=100.0(\mathrm{~s}) \quad t_{y}=60.0(\mathrm{~s}) \quad t_{z}=130.0(\mathrm{~s}) \\
& \theta_{x}=20.0(\mathrm{deg}) \quad \theta_{y}=80.0(\mathrm{deg}) \quad \theta_{z}=120.0(\mathrm{deg})
\end{aligned}
$$

Control gains $K_{r}$ and $K_{v}$ are scalar and given by

$$
K_{r}=0.1\left(1 / s^{2}\right) \quad K_{v}=0.1(1 / s)
$$

The parameters in the commanded reference trajectories of the two maneuvers have the following values.

$$
\begin{aligned}
& \omega=1.0(\mathrm{deg} / \mathrm{s}) \quad \theta_{0}=0.0(\mathrm{deg}) \\
& r_{m i}=10.0(\mathrm{~m}) \quad r_{m f}=0.0(\mathrm{~m}) \\
& t_{i}=200.0(\mathrm{~s}) \quad t_{f}=1200.0(\mathrm{~s})
\end{aligned}
$$

The three components of the chaser commanded thrust forces $T_{c}$ is limited to $8 N$. For this two maneuvers, $f_{\max }$ is dominated by the target thrust forces and estimated by

$$
f_{\max } \approx\left(\begin{array}{ccc}
T_{x} & T_{y} & T_{z}
\end{array}\right)^{T} / m_{t}
$$

and

$$
\left\|K_{r}^{-1} f_{\max }\right\|=0.112(m)
$$

The actual value of the maximum of $\|\lambda\|$ is slightly larger than $0.112 \mathrm{~m}$ because $f_{\max }$ includes only target thrust forces.

The time histories of the rendezvous maneuver with the chaser spacecraft approaching the target spacecraft along a spiral trajectory in the target spacecraft's in-track plan are shown in Figure 3 to Figure 8. Figure 4 shows that the value of the maximum of $\|\lambda\|$, when it converges, is $0.118 \mathrm{~m}$, which is slightly larger than $0.112 \mathrm{~m}$. The commanded thrust forces in in-track direction reaches the limit $(8 \mathrm{~N})$ during the first 60 seconds.

Figure 9 to Figure 14 is the time histories of the second rendezvous maneuver. The chaser spacecraft approaches the target spacecraft along a spiral trajectory in the target spacecraft's cross-track plans. The value of the maximum 
of $\|\lambda\|$ is $0.118 \mathrm{~m}$ also when it converges as shown in Figure 10. Figure 12 and Figure 13 show the chaser spacecraft approaches the target spacecraft in the in-track, radial plan and cross-track, radial plan. As we expected, the chaser spacecraft approaches the target spacecraft along a spiral trajectory. Figure 14 shows the commanded thrust forces calculated by the control algorithm to accomplish the desired rendezvous maneuver. The results show the excellent performance of the control system.

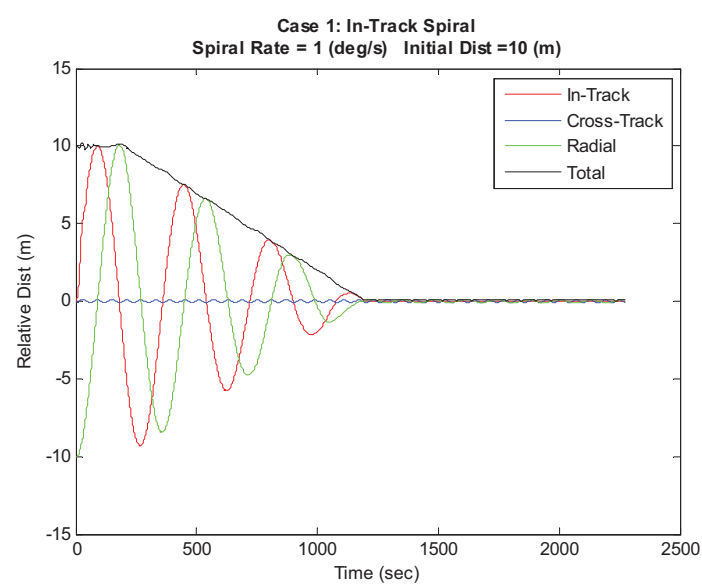

Figure 3. Case 1: Relative Distance

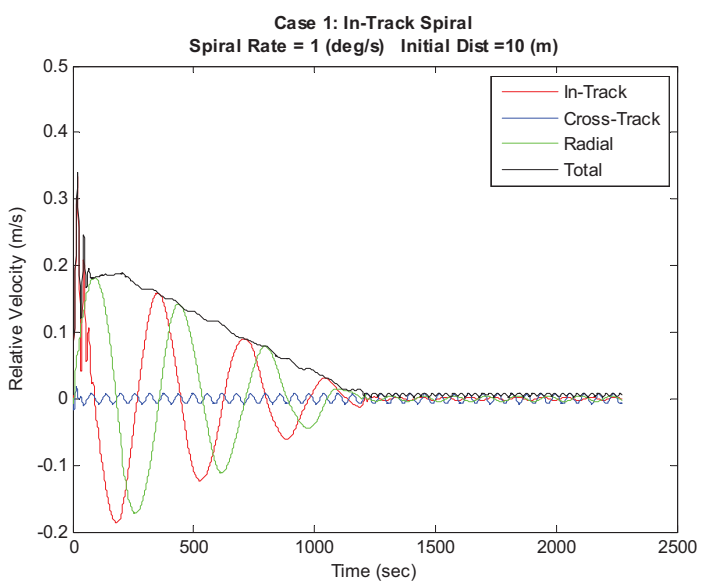

Figure 5. Case 1: Relative Velocity

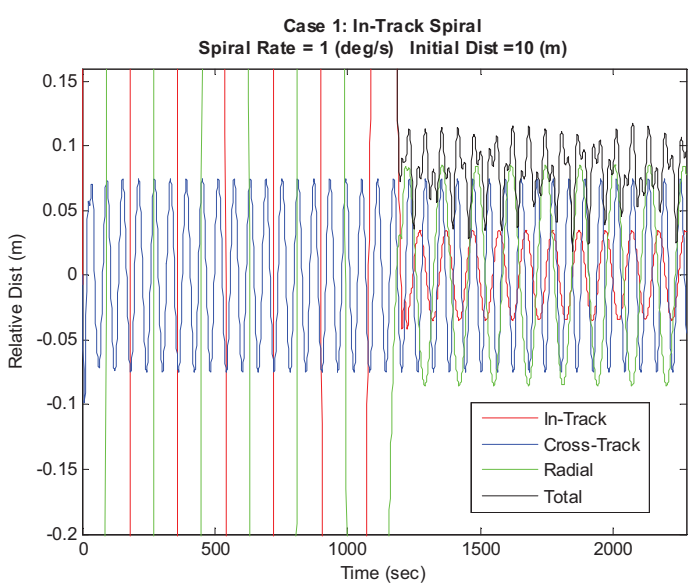

Figure 4. Case 1: Relative Distance (zoomed in)

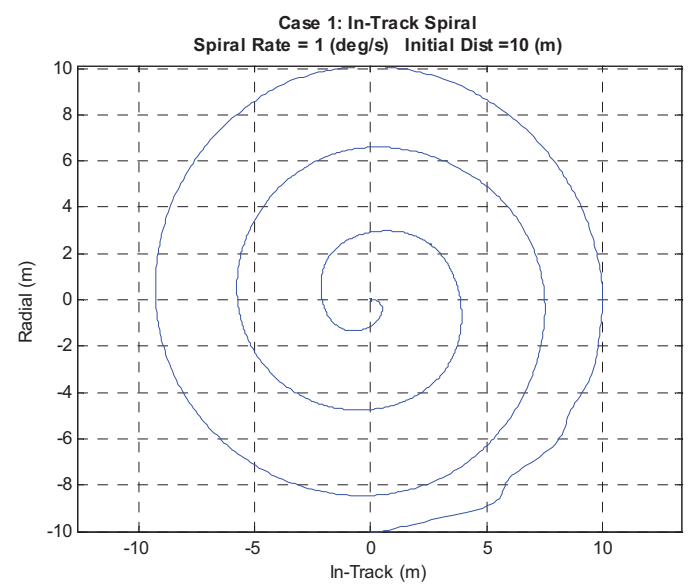

Figure 6. Case 1: Relative position in radial and intrack plan 


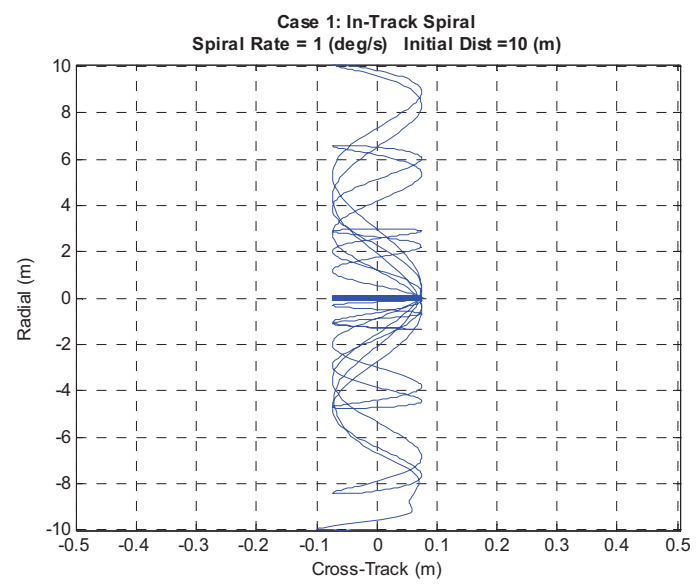

Figure 7. Case 1: Relative position in radial and cross-track plan

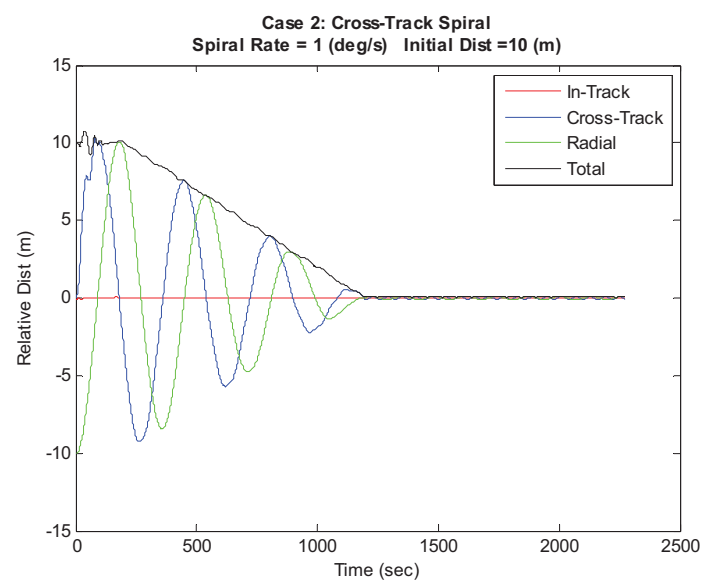

Figure 9. Case 2: Relative Distance

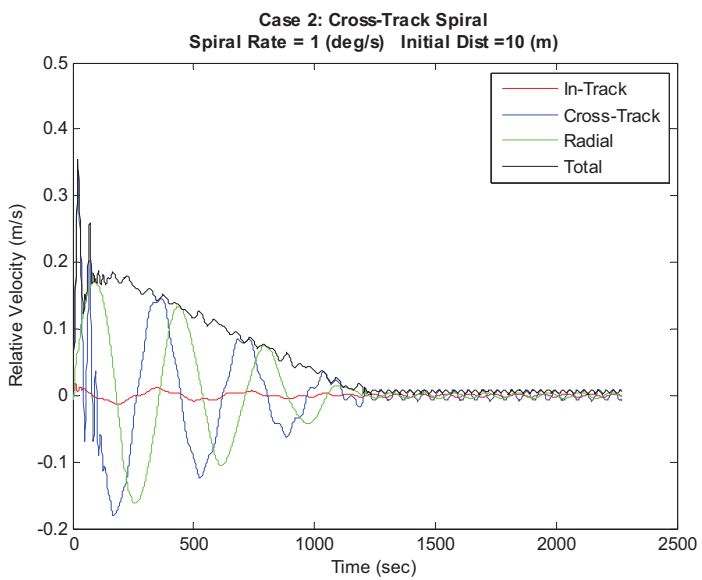

Figure 11. Case 2: Relative Velocity

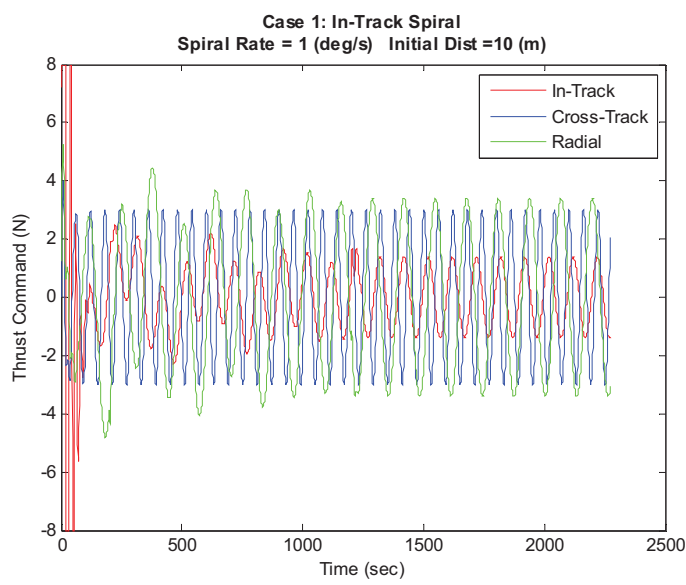

Figure 8. Case 1: Thrust forces command

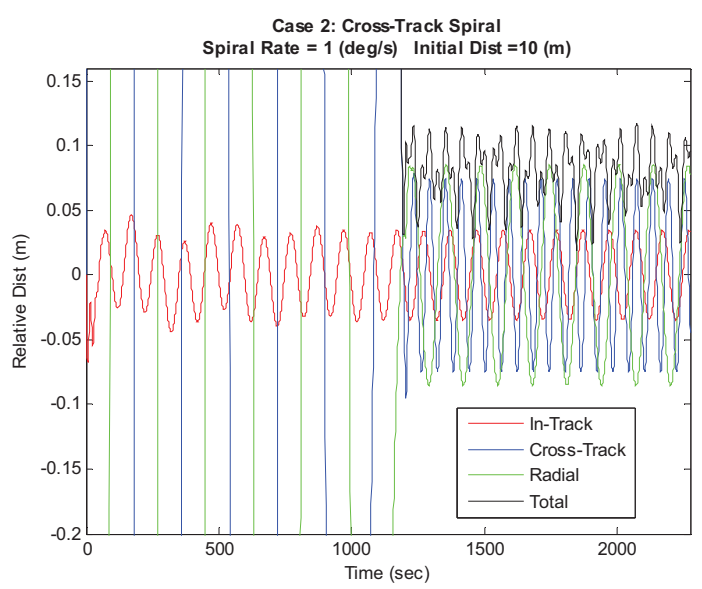

Figure 10. Case 2: Relative Distance (zoomed in)

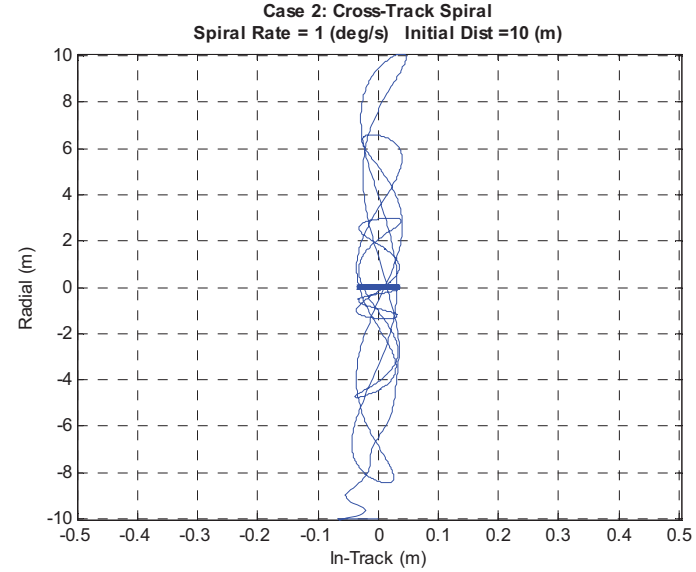

Figure 12. Case 2: Relative position in radial and intrack plan 


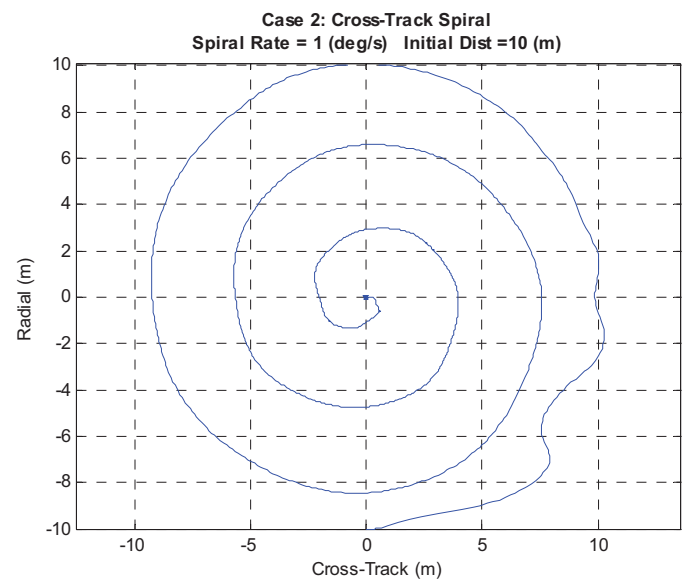

Figure 13. Case 2: Relative position in radial and cross-track plan

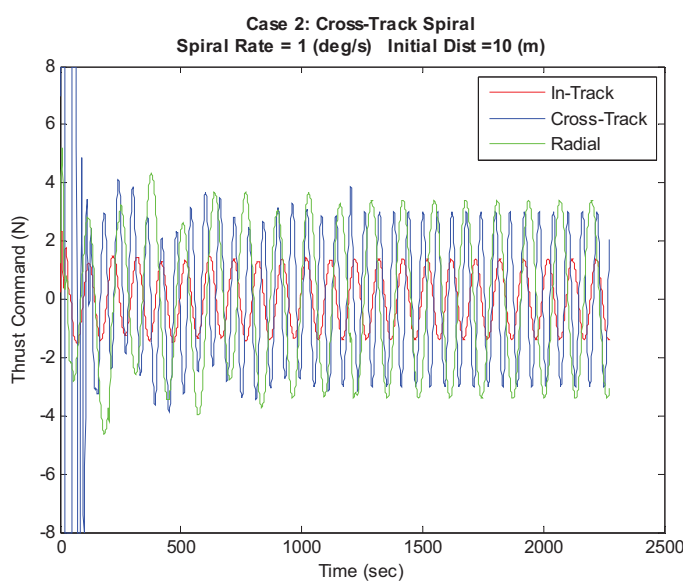

Figure 14. Case 2: Thrust forces command

\section{Conclusion}

A nonlinear trajectory control algorithm of rendezvous with maneuvering target spacecraft is developed in this paper. The disturbance forces on the chaser and target spacecraft and the thrust forces on the chaser spacecraft are considered in the analysis. The control algorithm developed in this paper uses the relative distance and relative velocity between the target and chaser spacecraft as the inputs. A general formula of reference relative trajectory of the chaser spacecraft to the target spacecraft is developed and applied to four different proximity maneuvers, which are in-track circling, cross-track circling, in-track spiral rendezvous and cross-track spiral rendezvous. The closedloop differential equations of the proximity relative motion with the control algorithm are derived. The tracking errors between the commanded relative trajectory and the actual relative trajectory are analyzed. The prediction of the tracking errors is obtained. Design examples are provided to show the implementation of the control algorithm. The simulation results show that the actual relative trajectory tracks the commanded relative trajectory tightly. The predicted tracking errors match those calculated in the simulation results.

\section{Acknowledgments}

The author would like to thank Mark Hutchinson and Arthur Bradley for their support and encouragement.

\section{References}

${ }^{1}$ Zhou, Bin, Lin, Zongli, and Duan, Guang-Ren, "Lyapunov Differential Equation Approach to Elliptical Orbital Rendezvous with Constrained Controls", Journal of Guidance, Control, and Dynamics, Vol. 34, No. 2, 2011, pp. 345-358.

${ }^{2}$ Karlgaard, C., and Schaub, H., "Adaptive Nonlinear Huber-Based Navigation for Rendezvous in Elliptical Orbit”, Journal of Guidance, Control, and Dynamics, Vol. 34, No. 2, 2011, pp. 388-402.

${ }^{3}$ Zanon, D., and Campbell, M., "Formation Optimal Maneuvers Under Inertial Attitude Dynamics", Journal of Spacecraft and Rockets, Vol. 47, No. 2, 2010, pp. 295-307.

${ }^{4}$ Yamanaka, K., and Ankersen, F., "New State Transition Matrix for Relative Motion on an Arbitrary Elliptical Orbit," Journal of Guidance, Control, and Dynamics, Vol. 25, No. 1, 2002, pp. 60-66.

${ }^{5}$ Carter, T. E., "State Transition Matrices for Terminal Rendezvous Studies: Brief Survey and New Example," Journal of Guidance, Control, and Dynamics, Vol. 21, No. 1, 1998, pp. 148-155. 